\title{
Provision of Chlamydia trachomatis screening in family planning clinics and emergency contraception in genitourinary medicine clinics: A collaborative cross-speciality survey
}

\author{
A W S Dale, BSc, RN, Clinical Governance Support Unit, Camden \& Islington CHS NHS Trust, St Pancras Hospital, London, \\ UK and North Thames Regional Audit Group for GU/HIV Medicine, London, UK; C Wilkinson, MFFP, Caldecot Centre, King's \\ College Hospital NHS Trust, London, UK; G E Forster, FRCOG, North Thames Regional Audit Group for GU/HIV Medicine, \\ London, UK and Ambrose King Centre, Royal London Hospital, London, UK; D Daniels, FRCP, North Thames Regional Audit \\ Group for GU/HIV Medicine, London, UK and Department of GU Medicine, West Middlesex University Hospital, Twickenham, \\ UK; M G Brook, FRCP, North Thames Regional Audit Group for GU/HIV Medicine, London, UK and Patrick Clements Clinic, \\ Central Middlesex Hospital, London, UK on behalf of the North Thames Regional Audit and Education Group for GU/HIV \\ Medicine
}

Correspondence: A W S Dale, Clinical Governance Resource Group, South East London Shared Services Partnership, GKT Department of General Practice and Primary Care, 3rd Floor Weston Education Centre, Cutcombe Road, London SE5 9RJ, UK. E-mail: Adam.Dale@kcl.ac.uk

(Accepted $4^{\text {th }}$ August 2002)

The Journal of Family Planning and Reproductive Health Care 2002: 28(4): 185-188

\begin{abstract}
Two surveys were undertaken to review (1) provision of Chlamydia trachomatis screening by family planning (FP) clinics in the London region and (2) access to emergency contraception (EC) from genitourinary medicine (GUM) clinics within the former North Thames region. The findings from the first survey suggest that there is insufficient screening (and treatment) in vulnerable groups attending FP clinics. Results from the second survey show that hormonal EC is widely available from within GUM clinics, and those clinics also provide a range of other contraception services. However, these details may not be widely recognised either by policymakers or the general public.
\end{abstract}

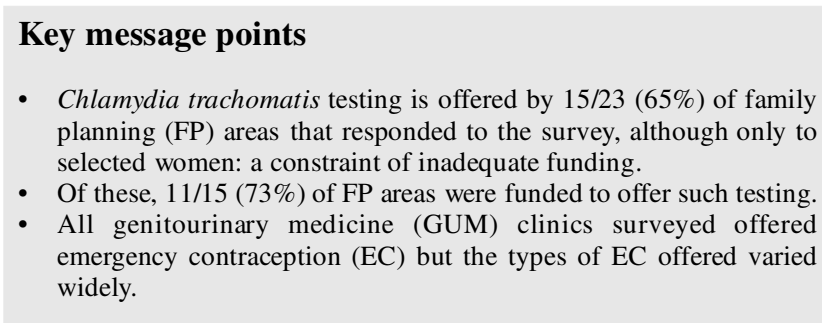

\section{Introduction}

Genitourinary medicine (GUM) and family planning (FP) clinics provide a significant portion of the sexual health care available within the UK's National Health Service (NHS). GUM is mainly concerned with sexually transmitted infections, including Chlamydia trachomatis. GUM clinics also provide contraceptive advice, especially emergency contraception (EC). FP clinics offer mainly contraception provision, including EC, but some clinics undertake screening for chlamydial infection. If left untreated $C$. trachomatis infection is associated with a range of adverse sequelae, including pelvic inflammatory disease. These infections are costly both in terms of the cost of human suffering and the cost of treatment for the NHS. ${ }^{1}$ Early diagnosis and treatment minimises these costs. There were three methods of EC used in the UK at the time of the study: progestogen-only emergency contraception (POEC), combined hormonal emergency contraception (CHEC) Yuzpe method, and the copper-containing intrauterine device (IUD). ${ }^{2}$ The boundary, which may once have existed between these services, is becoming less distinct with greater co-operation between the professionals who work within each service. ${ }^{3}$ This co-operation has been nurtured by an appreciation of the overlapping needs of the clients who access these services. ${ }^{4-8}$

\section{Study objectives and design}

The objectives of the present study were two-fold. (1) To evaluate how FP clinics in London had incorporated C. trachomatis screening and treatment, specifically prior to procedures such as coil fitting, and in offering opportunistic screening of service users or targeted testing of symptomatic women. (2) To gauge the extent GUM clinics in North Thames were offering EC. The study comprised two cross-sectional surveys.

\section{Study participants and methods}

In Autumn 2000, two regional surveys were conducted within London and the former North Thames regions (1) to review the provision of chlamydia screening within FP clinics located in the London region and (2) to evaluate the access to EC from GUM clinics in the former North Thames region. Both surveys were co-ordinated by the North Thames Regional Audit Group for GU/HIV Medicine and had the support and encouragement of medical staff at the Faculty of Family Planning and Reproductive Health Care (FFPRHC) of the Royal College of Obstetricians and Gynaecologists (RCOG).

Both surveys used self-completed questionnaires to collect information. The questionnaires used to survey provision of chlamydia screening within FP clinics in London were mailed to each of 28 consultants and senior community medical officers (SCMOs) in FP with instructions that they answer questions on behalf of clinics to reflect this provision. Only those doctors whose clinics offered screening were asked to complete the remainder of the survey.

The questionnaires used to survey provision of EC within GUM clinics were mailed to the lead consultant, or 
consultant designated to participate in regional audit, in each of 33 GUM clinics in North Thames, with similar instructions to supply answers which reflected clinic practice. Only those doctors whose GUM clinics offered EC were asked to complete the remainder of the survey.

\section{Results}

Provision of C. trachomatis screening in FP clinics

Completed survey questionnaires were returned by 22 consultants or SCMOs, with one doctor returning two forms to reflect practice within the two FP areas under his jurisdiction (response rate: 23/29 $=79 \%$ ). Data are presented in terms of FP areas. FP areas were used in preference to single FP clinics since there may be several FP clinics within one area, but all operate under the same lead doctor and clinical guidelines. Surveying each FP clinic would have resulted in the same lead doctor completing numerous forms with identical information. The numbers of clinics within each FP area were not requested, however the $29 \mathrm{FP}$ areas covered the whole of London region.

Fifteen FP areas $(15 / 23,65 \%)$ offered screening for C. trachomatis and eight did not. One of the eight FP areas not offering screening had offered this service for a 1-year trial period (the respondent did not indicate the dates when this trial commenced or finished). However, none of the women offered screening during the trial period gave informed consent to testing. Testing was therefore not possible and the service was withdrawn.

The following results apply only to those FP areas offering C. trachomatis screening. Table 1 gives details of which women were offered such screening. No FP areas offered routine screening to all women attending their area FP clinics. A total of 12/15 FP areas used swabs to collect urethral and endocervical material or endocervical material alone for screening. Screening using nucleic acid amplification techniques (NAAT) were offered at four FP areas $(27 \%)$, three using first-pass urine (FPU/early morning sample) and one using material collected by swab. Enzyme-linked immunoassay (EIA) was employed by clinics in $11 \mathrm{FP}$ areas $(73 \%)$.

Eleven FP areas $(11 / 15,73 \%)$ were funded to provide C. trachomatis screening as a routine service provision. The other four used other sources of funding (not specified). One FP area $(1 / 15,7 \%)$ offered chlamydial screening to male partners of women found to have $C$. trachomatis infection. All other FP areas referred such men to other agencies, usually the GUM service. Three FP areas (3/15,
$20 \%$ ) were funded to provide treatment for women; one of these was only funded to offer antibiotic prophylaxis (drug not stated) to women prior to fitting of an intrauterine device (IUD).

\section{Provision of EC by GUM clinics}

Completed questionnaires were returned by 25/33 GUM clinics returned, giving a $76 \%$ response rate. The results below use the number of responders as denominator.

FP and contraception services. Consultants in 19/25 clinics (76\%) stated that their clinics offered FP services. Fourteen of these clinics $(74 \%)$ offered the service within the routine GUM clinic, with 11 of these $(79 \%)$ also offering specific appointment/walk-in FP clinics. Four clinics (4/19, 21\%) offered FP in designated young people's clinics only.

All 25 GUM clinics surveyed offered some form of EC. All GUM clinics provided condoms to their clients. Table 2 shows which health care professionals offered the service. A total of 19/25 clinics (76\%) offered more comprehensive FP services. Table 3 outlines the range of methods of EC offered.

IUD EC. A total of 8/25 (32\%) clinics offered this method. Of the eight clinics providing IUD EC, five clinics (63\%) had written guidelines for providing IUD EC.

Progestogen-only method: Levonelle 2. A total of 24/25 clinics offered POEC (96\%). Eighteen clinics (75\%) had guidelines, with one clinic in the process of drafting a guideline. Three clinics $(3 / 24,13 \%)$ obtained written consent prior to administration. Twenty clinics $(83 \%)$ did not prescribe antiemetics with Levonelle 2, the other four prescribing them only for women who developed symptoms of nausea with Levonelle 2 or other progestogen-only contraception, or who were anxious about this. Domperidone $20 \mathrm{mg}$ and prochlorperazine $5 \mathrm{mg}$ were the anti-emetics of choice (one unit did not indicate which anti-emetic was prescribed).

Combined oestrogen and progestogen: Yuzpe method. A total of $12 / 25$ clinic offered CHEC (48\%). Nine clinics $(9 / 12,75 \%)$ had written guidelines. Seven clinics $(7 / 12$, $58 \%$ ) routinely prescribed anti-emetics with the Yuzpe method, with four clinics prescribing sometimes, and one did not prescribe. Prochlorpererazine, domperidone, cyclizine and metoclopramide were the anti-emetics of choice prescribed.

Table 1 Women to whom C. trachomatis testing was offered in FP areas offering testing and responding to the survey

\begin{tabular}{ll}
\hline & $\begin{array}{l}\text { FP areas offering } \\
\text { screening }(\mathrm{n}=15)\end{array}$ \\
\hline $\begin{array}{l}\text { Women with symptoms and/or signs indicative } \\
\text { of infection, or based on clinical/sexual history }\end{array}$ & $11 / 15(73)^{\mathrm{b}}$ \\
$\begin{array}{l}\text { Pre-IUD/IUS according to protocol criteriac } \\
\text { (risk factors identified) }\end{array}$ & $8 / 15(53)$ \\
$\begin{array}{l}\text { Pre-TOP referral } \\
\text { Pre-IUD/IUS in women }<25 \text { years }\end{array}$ & $5 / 15(33)$ \\
$\begin{array}{l}\text { Pre-IUD/IUS in nulliparous women } \\
\text { Women of male partners with symptoms and/or signs }\end{array}$ & $2 / 15(13)$ \\
indicative of infection, or based on clinical/sexual history & $2 / 15(13)$ \\
\hline
\end{tabular}

aparticipants could select more than one option.

bPercentage values are given in parentheses.

'Protocol criteria: more than one sexual partner in the past 12 months, partner change, recent history of STI.

FP, Family planning; IUD, intrauterine device; IUS, intrauterine system; STI, sexually transmitted infection; TOP, termination of pregnancy. 
Table 2 Which health care professionals offered FP and contraception? Clinics $(\mathrm{n}=19) \quad$ Percentage $(\%)$

\begin{tabular}{lll}
\hline $\begin{array}{l}\text { GUM clinic doctor with special } \\
\text { interest in FP }\end{array}$ & 13 & 68 \\
$\begin{array}{l}\text { GUM nurse with special } \\
\text { interest in FP }\end{array}$ & 11 & 58 \\
Designated FP doctor & 8 & 42 \\
Designated FP nurse & 8 & 42 \\
GUM clinic doctors & 2 & 10 \\
Local GP with experience in & 1 & 5 \\
GUM and FP & 1 & 5 \\
Clinical assistants in sexual health &
\end{tabular}

aThe meaning of the term sexual health is unclear in this context. FP, Family planning: GP, general practitioner; GUM, genitourinary medicine.

Table 3 Methods of emergency contraception offered by GUM clinics $^{a}$

\begin{tabular}{lll}
\hline & Clinics $(\mathrm{n}=25)$ & Percentage $(\%)$ \\
\hline Levonelle 2 and Yuzpe & 9 & 36 \\
Levonelle 2 & 7 & 28 \\
IUD and Levonelle 2 & 6 & 24 \\
IUD, Levonelle 2 and Yuzpe & 2 & 8 \\
Yuzpe & 1 & 4 \\
Totals & 25 & 100 \\
\hline
\end{tabular}

a A total of 25 clinics responded to the survey.

GUM, Genitourinary medicine; IUD, intrauterine device.

\section{Discussion}

The survey of provision of $C$. trachomatis screening in FP clinics in the London region shows that only two-thirds of FP clinic areas were offering such screening to their patients. EIA was the main screening method, in preference to the newer NAAT, although studies have shown demonstrable cost benefits of the latter. ${ }^{9}$ Of those FP areas providing screening, most were funded to provide targeted screening only, for example, women being referred for termination of pregnancy (TOP). These policies will inevitably result in many asymptomatic infections remaining undiagnosed and untreated. There is evidence that many clients referred to GUM clinics by FP clinics do not attend. ${ }^{10,11}$ It is of concern that only one-fifth of the FP clinic areas offering screening were also funded to offer treatment for $C$. trachomatis infection.

A major shortcoming of the present survey was that it did not request information about the number of clinics within each FP area or information about the numbers of women seen or local population size. Therefore, although results indicate that $C$. trachomatis testing was offered by $15 / 23(65 \%)$ of FP areas surveyed, this might not reflect $65 \%$ of the population located within these FP areas, with actual screening coverage being lower or higher. This information is important in order to understand the future funding needs of FP services if they are to be able to offer screening for C. trachomatis

The survey of GUM clinics showed that all clinics were offering EC to clients and that three-quarters of clinics offered other FP service. Levonelle 2 was the most widely prescribed method, offered by all but one GUM clinic. Only one-half of GUM clinics surveyed prescribed the Yuzpe method, possibly reflecting the lower efficacy and greater risk of side effects of this method compared with Levonelle $2 .^{12}$ Only one-third of GUM clinics offer IUD EC, which may be due to a lack of time in consultations ${ }^{13}$ or lack of expertise. Provision of EC was largely in accordance with the guidelines issued by the FFPRHC of the RCOG in use at the time of the survey. ${ }^{2}$

This survey did not ask for information about the funding of EC provision in GUM clinics, although this information was requested in the survey of FP clinics regarding provision of $C$. trachomatis screening/treatment. This prevented comparison of the relative funding positions for these service provisions from being made.

Demonstrable health care benefits of offering a FP service within the GUM clinic have been published, ${ }^{14,15}$ illustrating the importance of offering contraception to women who do not access conventional sources of FP services. This contribution has, on occasion, been overlooked. ${ }^{8,16}$

The fairly high non-responder rates for both surveys perhaps reflect the limited time busy clinicians have to participate in surveys of this type. The non-responder rate for the GUM survey may perhaps be a reflection of 'survey fatigue', with units requested to participate in at least four surveys and audits per year, as part of the North Thames Regional Audit Programme. Given the increasing clinical governance work clinical specialties are obliged to undertake, including clinical audit, a similar effect might be attributable to the FP service. Nonetheless, these surveys provide some useful information regarding provision of C. trachomatis screening in FP clinics and access to FP and EC in GUM clinics in the London and North Thames regions.

\section{Acknowledgements}

For the survey of FP clinics, we thank Imelda O'Brien at the FPA and Dr Connie Smith at the FFPRHC for help and advice planning this survey. We also thank the consultants and SCMOs in the London region who took part in the survey.

For the survey of GUM clinics, we thank Catherine Godfrey for use of an adapted questionnaire originally conceived by Ms Godfrey. We also thank the consultants working in the GUM clinics in North Thames who took part in the survey.

We thank the London Specialist Commissioners Advisory Group and Camden \& Islington CHS NHS Trust for funding the work of the Regional Audit Group.

GF, DD and GB conceived the survey. CW and GF developed the questionnaires with contributions from DD and GB. AD administered the survey, cleaned and analysed the data. All authors contributed to the drafting of the article.

\section{Statements on funding and competing interests}

Funding. None identified.

Competing interests. None identified.

\section{References}

1 Clinical Effectiveness Group. Authors: Horner PJ, Caul EO. National guideline for the management of Chlamydia trachomatis genital tract infection. Sex Transm Inf 1999; 75(Suppl. 1): S4-S8.

2 Faculty of Family Planning and Reproductive Health Care. Recommendations for clinical practice: emergency contraception. London: Clinical and Scientific Committee, Faculty of Family Planningand Reproductive Health Care, 2000.

3 Ward H, Kubba A, Bradbeer C, et al. Consensus workshop on sexually transmitted diseases and contraception: sexual health promotion and service delivery. Faculty of Family Planning and Reproductive Health Care; Faculty of Public Health Medicine; MSSVD, 1995.

4 Mann MC, Radcliffe KW, Basarab M. Knowledge of emergency contraception amongst female patients attending a department of genitourinary medicine. Br J Fam Plann 1999; 25: 58-62.

5 Nanthakumaran H, Sullivan AK, Boag FC. An audit of emergency contraception: a look at patient characteristics and the effects of a 
consultation proforma. Int J STD AIDS 1998; 9: 48-50.

6 Simms, I, Hopwood J, Mallinson H, et al. Changing screening strategies for genital chlamydia in family planning clinics: a good public health strategy? Eur J Contracept Reprod Health Care 2000; 5: 91-95.

7 James NJ, Hughes S, Ahmed-Jushuf I, et al. A collaborative approach to management of chlamydial infection among teenagers seeking contraceptive care in a community setting. Sex Trans Inf 1999; 75: 156-161.

8 Forster GE, Dale AWS, Daniels D, et al. Family planning provision in GUM clinics. Br J Fam Plann 2000; 26: 244.

9 Howell MR, Quinn TC, Braithwaite W, et al. Screening women for Chlamydia trachomatis in family planning clinics: the cost effectiveness of DNA amplification assays. Sex Transm Dis 1998; 25: $108-117$.

10 Wilkinson C, Massil H, Evans J. An interface of chlamydia testing by community family planning clinics and referral to hospital genitourinary medicine clinics. Br J Fam Plann 2000; 26: 206-209.

11 Vanhegan G, Wedgwood A. Young people's understanding of safer sex and their attitude to referral for STI screening - two audits from London Brook Advisory Centres. Br J Fam Plann 1999; 25: 22-23.

12 Ho PC. Emergency contraception: methods and efficacy. Curr Opin Obstet Gynecol 2000; 12: 175-179.

13 Reuter S. Barriers to use of IUDs as emergency contraception. $\mathrm{Br} \mathrm{J}$ Fam Plann 1999; 25: 61-68.

14 Masters L, Nicholas H, Bunting P, et al. Family planning in genitourinary medicine: an opportunistic service? Genitourin Med 1995; 71: 103-105.

15 Carlin EM, Russell JM, Sibley K, et al. Evaluating a designated family planning clinic within a genitourinary medicine clinic. Genitourin Med 1995; 71: 106-108.

16 Bardsley M, Morgan D, Jacobsen B. Estimating the balance of general practice versus family planning clinic coverage of contraception services in London. Br J Fam Plann 2000; 26: 21-25.

\section{Oves $^{\circledR}$ contraceptive cap: Short-term acceptability, aspects of use and user satisfaction}

Judith Roizen, PhD, School of Sport and Health Sciences, University of Exeter, Exeter, UK; Sue Richardson, MB ChB, MFFP, Consultant in Family Planning and Reproductive Health Care, Keighley Health Centre, Keighley, UK; John Tripp, MD, FRCPCH, Senior Lecturer, School of Sport and Health Sciences, University of Exeter, Exeter, UK; Hilary Hardwicke, MA, School of Sport and Health Sciences, University of Exeter, Exeter, UK; Tran Quang Lam, MA, Personnel and Training Department, National Committee for Population and Family Planning, Hanoi, Vietnam

Correspondence: Dr Judith Roizen, 3 Pennsylvania Park, Exeter EX4 6HB, UK. Tel: +44 (0) 1392272986. Fax: +44 (0) 1392421360. E-mail: roizen_caston@compuserve.com

Family Planning and Reproductive Health Research Network Principal Investigators: J Bateman (Portsmouth), D Booker (Newport), S Carr (Glasgow), H Cooling (Bristol), T Laverty (Bath), C Nash (Norwich), S Richardson (West Yorkshire - Lead Investigator), P Saxty (York), R Tolcher (Southampton), C Tupper (Cumbria) in collaboration with A Kubba (London)

(Accepted $14^{\text {th }}$ August 2002)

The Journal of Family Planning and Reproductive Health Care 2002: 28(4): 188-191

\begin{abstract}
Objective. To assess the short-term acceptability, aspects of use and user satisfaction with the Oves ${ }^{\circledR}$ cap.

Design, setting and subjects. A multicentre observational study, commissioned by Veos Ltd, manufacturers of the Oves ${ }^{\circledR}$ cap, was carried out by the UK Family Planning and Reproductive Health Research Network in collaboration with the Institute of Population Studies, University of Exeter, Exeter, UK. Women from ten Network centres and one collaborating centre were invited to participate. Following an assessment by vaginal examination women were fitted with the cap and taught self-fitting by a doctor. The women were asked to use the cap six times in 8 weeks. Participants were asked to complete four questionnaires on various aspects of cap use including Likert-type measures and open-ended questions on experiences with the cap. Doctors were asked to complete a first visit and follow-up questionnaires. Women were self-selected clients in the participating centres. Women aged 18 years and over, gynaecologically healthy, using hormonal contraception or sterilised were eligible for the study. Thirty-five women were enrolled and fitted with the cap; 20 chose to participate in the study.

Main outcome measures. Ease of fitting and removal of the cap expressed in structured and open-ended questions by both cap users and doctors; satisfaction of women and partners with the cap, measured by desire to use the cap in the future and by premature withdrawal from the trial.
\end{abstract}

Results. Twenty women used the cap on a total of 84 occasions. Four women completed the trial of six uses. While most doctors did not have difficulty with fittings or removals, 10/20 Oves ${ }^{\circledR}$ cap users reported some difficulty in fitting it over the cervix and 12 reported some difficulty removing it in the first three uses. Fewer women had difficulty in fitting in uses 4-6 but nearly half continued to have some difficulty with removals.

Conclusions. Few women indicated that they would use the cap in the future. However, most women were satisfied with their current method of contraception. The study raises the question whether women using non-barrier methods of contraception and satisfied with their current method of contraception are the appropriate target recruits for a trial such as this, even in the absence of robust efficacy data.

\section{Key message points}

- The Oves ${ }^{\circledR}$ cap is a disposable silicone cap that can be left in situ for 72 hours.

- Ease of fitting of the Oves ${ }^{\circledR}$ cap increased with increased use, but some women continued to experience difficulty in removing the cap.

Women recruited to this acceptability study were already using nonbarrier contraception.

- The Oves ${ }^{\circledR}$ cap may offer an additional choice of contraception for some women, when UK efficacy data is available. 